Acta Crystallographica Section A

Foundations of Crystallography

ISSN 0108-7673

Received 1 October 2007

Accepted 9 November 2007

\section{Report on a project on three-dimensional imaging of the biological cell by single-particle X-ray diffraction. Addendum}

\author{
David A. Shapiro \\ Advanced Light Source, Lawrence Berkeley National Laboratory, 1 Cyclotron Road, Berkeley, \\ CA 94720, USA. Correspondence e-mail: dashapiro@lbl.gov
}

\begin{abstract}
In the paper by Sayre [Acta Cryst. (2008), A64, 33-35], a proposal is made to use stereoscopy as a short-term means of overcoming the primarily technological hurdles involved in three-dimensional imaging of the biological cell by soft $\mathrm{X}$-ray diffraction microscopy. This addendum provides a broader perspective on the techniques used by this rapidly maturing community to investigate structural problems in the biological and material sciences.
\end{abstract}

(C) 2008 International Union of Crystallography Printed in Singapore - all rights reserved tion will allow for the three-dimensional visualization of every unit cell in the crystal and provide valuable information for crystal growth studies.

The large dose required for single-particle imaging implies that the resolution of such images will be limited by the radiation damage to sensitive particles for which there is only a single copy. This is particularly important for isolated biological cells that are structurally unique. It has been suggested that this limit can be overcome in the case that many identical copies of the single particle are available. Protein molecules, particularly those which resist crystallization, are structurally identical and available sequentially in time. The challenge then becomes to record adequate diffraction data from many individual proteins before radiation damage is manifest in each one. There have been two methods proposed to achieve this. The first, which was proposed by Neutze and provides perhaps the strongest justification for building the Linac Coherent Light Source at SLAC, requires the ultrabright and ultra-short X-ray pulses to be delivered by an X-ray free-electron laser (Neutze et al., 2000). This method, now called flash imaging, uses a single few-femtosecond X-ray pulse per protein molecule. Such short pulses will allow for the collection of atomic resolution diffraction data from a single molecule before the pulse destroys the molecule. The full three-dimensional set of Fourier magnitudes can be acquired by recording such diffraction patterns from many randomly oriented proteins. Post-processing can then orient and average the recorded patterns. Flash imaging of test objects was recently demonstrated by Chapman using the VUV-FEL FLASH at DESY (Chapman, Barty, Bogan et al., 2006).

The second method aims for lower resolution, adequate for seeing secondary protein structure, but only requires a thirdgeneration synchrotron. This recently proposed serial protein crystallography method establishes a situation which is effectively identical to having a single protein in a continuously running coherent X-ray beam thus allowing for collection of its single-particle diffraction pattern (Spence \& Doak, 2004; 
Spence et al., 2005). The proteins are delivered to the X-ray beam as a collimated particle beam whose velocity is such that each protein molecule is only briefly exposed to radiation and therefore is not damaged. The orientation of the proteins is provided by the interaction between a tightly focused laser field and the protein's induced dipole moment. The resolution of this method is limited by the total exposure time and the degree of particle alignment. Recent simulations indicate that a resolution of $7 \AA$ can be attained with reasonable exposure times at currently available X-ray sources (Starodub et al., 2007). Therefore, serial protein crystallography should provide a high-throughput means of structure determination that is competitive with cryoelectron microscopy. Preliminary experiments aimed at optimizing the X-ray illumination and particle source are ongoing at the Advanced Light Source (Weierstall et al., 2007).

The challenges faced by the cell-imaging project are formidable. However, the use of anti-contamination devices, similar to those used in cryoelectron microscopes, will greatly improve the long-term stability of the specimen ice layer. Also, a move to harder $\mathrm{X}$-rays, in the $2-3 \mathrm{keV}$ range, will bring the $4 \mu \mathrm{m}$ cell into the weak scattering limit. The absorption contrast will be greatly reduced but diffraction imaging produces absorption and phase-contrast images simultaneously so the imaging capability will not be compromised. The prospect of imaging frozen-hydrated cells in three dimensions at $10 \mathrm{~nm}$ resolution is the basis of a proposal to build a new dedicated X-ray beamline at the Advanced Light Source (ALS). The optimized source will have orders-ofmagnitude more coherent flux and will reduce total exposure times for three-dimensional imaging from hours to minutes. The work done at the ALS is a small part of a broad and expanding community of researchers with existing and proposed programs at the APS, ESRF, SLAC, Diamond, DESY and SPring- 8 . These researchers are developing a tool that will continue to contribute significantly to the fields of cell biology, molecular biology, materials/nanoscience for many years to come.

\section{References}

Barty, A., Marchesini, S., Chapman, H. N., Cui, C., Howells, M. R., Shapiro, D. A., Minor, A. M., Spence, J. C. H., Weierstal, U., Noy, A., Hau-Riege, S. P., Artyukhin, A. B., Baumann, T., Willey, T., van Buuren, T. et al. (2007). http://arXiv.org/abs/0708.4035v1.

Chapman, H. N., Barty, A., Beetz, T., Cui, C., He, H., Howells, M. R., Jacobsen, C., Noy, A., Shapiro, D., Spence, J. C. H., Hau-Riege, S. P., Rosen, R. \& Weierstall, U. (2006). J. Opt. Soc. Am., A23, $1179-1200$.

Chapman, H. N., Barty, A., Bogan, M. J., Boutet, S., Frank, M., HauRiege, S. P., Marchesini, S., Woods, B. W., Bajt, S., Benner, H., London, R. A., Plonjes, E., Kuhlmann, M., Treusch, R., Dusterer, S. et al. (2006). Nat. Phys. 2, 839-843.

Miao, J., Charalambous, P., Kirz, J. \& Sayre, D. (1999). Nature (London), 400, 342-344.

Miao, J., Chen, C., Song, C., Nishino, Y., Kohmura, Y., Ishikawa, T., Ramunno-Johnson, D., Lee, T. \& Risbud, S. (2006). Phys. Rev. Lett. 97, 215503.

Miao, J., Hodgson, K. O., Ishikawa, T., Larabell, C. A., LeGros, M. A. \& Nishino, Y. (2003). Proc. Natl Acad. Sci. USA, 100, 110-112.

Neutze, R., Wouts, R., van der Spoel, D., Weckert, E. \& Hajdu, J. (2000). Nature (London), 406, 752-757.

Pfeifer, M. A., Williams, G. J., Vartanyants, I. A., Harder, R. \& Robinson, I. K. (2006). Nature (London), 422, 63-66.

Robinson, I. K., Vartanyants, I. A., Williams, G. J., Pfeifer, M. A. \& Pitney, J. A. (2001). Phys. Rev. Lett. 87, 195505.

Sayre, D. (1980). Imaging Processes and Coherence in Physics, edited by M. Schlenker, pp. 229-235. Berlin: Springer.

Sayre, D. (2008). Acta Cryst. A64, 33-35.

Shapiro, D., Thibault, P., Beetz, T., Elser, V., Howells, M., Jacobsen, C., Kirz, J., Lima, E., Miao, H., Neiman, A. \& Sayre, D. (2005). Proc. Natl Acad. Sci. USA, 102, 15343-153465.

Spence, J. C. H. \& Doak, R. B. (2004). Phys. Rev. Lett. 92, 198102.

Spence, J. C. H., Schmidt, K., Wu, J. S., Hembree, G., Weierstall, U., Doak, B. \& Fromme, P. (2005). Acta Cryst., A61, 237-245.

Starodub, D., Rez, P., Hembree, G., Howells, M. R., Shapiro, D. A., Chapman, H. N., Fromme, P., Schmidt, K., Weierstall, U., Doak, R. B. \& Spence, J. C. H. (2007). http://arXiv.org/abs/0706.3427v1.

Thibault, P., Elser, V., Jacobsen, C., Shapiro, D. \& Sayre, D. (2006). Acta Cryst. A62, 248-261.

Weierstall, U., Doak, R. B., Spence, J. C. H., Starodub, D., Shapiro, D. A., Kennedy, P., Warner, J., Hembree, G., Fromme, P. \& Chapman, H. N. (2007). http://arXiv.org/abs/physics/0701129v1. 